Article

\title{
Cancer screening information at community health fairs: What the participants do with information they receive
}

\author{
Erica Monrose, Jessica Ledergerber, Derrick Acheampong, Lina Jandorf \\ Icahn School of Medicine of Mount Sinai, New York, NY, USA
}

\begin{abstract}
Significance for public health
Cancer screening adherence is an important public health issue, especially in poor and racial minority communities that bear a greater cancer mortality burden. As cancer prognosis is closely associated with stage at diagnosis, sustained efforts must be made to ensure that members of these communities are equipped with knowledge about life saving cancer screening interventions. This study looks at how Community Health Fairs can be effective tools to disseminate cancer prevention information in poor and racial minority communities, which have traditionally faced many barriers to screening. Understanding the utility of health fairs in removing knowledge gaps and information barriers is of importance in the development of targeted and robust cancer prevention efforts in underserved communities. This study describes how participants share cancer prevention materials received at health fairs.
\end{abstract}

\section{Abstract}

Background. To assess participants' reasons for seeking cancer screening information at community health fairs and what they do with the information they receive.

Design and methods. Mixed quantitative and qualitative approach was used. Community health fairs are organized in underserved New York City neighbourhoods. From June 14, 2016 to August 26, 2016, cancer prevention tables providing information about various cancer screenings were established at 12 local community health fairs in New York City. In-person and follow up telephone surveys assessing interest in the cancer prevention table, personal cancer screening adherence rates, information-sharing behaviours and demographic variables have been taken into account. Statistical analyses were performed using IBM SPSS 22.0: frequencies, descriptive, cross tabulations. All qualitative data was coded by theme so that it could be analysed through SPSS. For example, Were you interested in a specific cancer? may be coded as 2 for yes, breast cancer.

Results. One hundred and sixteen patrons participated in the initial survey. Of those, $88(78 \%)$ agreed to give their contact information for the follow-up survey and 60 follow-up surveys were completed (68\%). Of those who reported reading the material, $45 \%$ shared the information; $15 \%$ subsequently spoke to a provider about cancer screenings and $40 \%$ intended to speak to a provider.

Conclusions. Participants disseminated information without prompting; suggesting the reach of these fairs extends beyond the people who visit our table. Future studies should look at whether patrons would share information at higher rates when they are explicitly encouraged to share the information.

\section{Introduction}

Insight into how community health fair participants use cancer prevention and screening information they receive is of critical importance given that health fairs normally take place in low income and racial minority communities, communities which have historically reported the lowest rates of screening and highest rates of mortality from preventable cancers. ${ }^{1,2}$ Despite a trending decrease in cancer mortality overall in the United States, black Americans still suffer a $25 \%$ higher cancer mortality compared to their white counterparts and cancer continues to be the leading cause of death for non-white Hispanics in this country. ${ }^{3-5}$

Since cancer mortality is associated with early detection, addressing screening behaviours in underserved and racial minority communities is of interest. However, these communities face barriers to screening. For black Americans, these barriers include lack of knowledge or awareness of cancer screenings, lack of access to general preventive health care services, institutional or system barriers, socioeconomic status, language barriers, immigrant status, and cultural beliefs. ${ }^{6}$ Similarly, among non-White Hispanics, researched barriers include cost, lack of information on where to get services, wait time for medical appointments, transportation and language services. ${ }^{1}$ Health fairs can potentially reduce barriers and thereby mitigate cancer mortality in these already burdened communities.

The key barrier addressed in this study was lack of awareness about preventive screening measures and guidelines via the use of health fairs. At our organized health fairs, we were able to educate community members about the benefits of screening, different screening methods, recommended screening schedules, and the importance of screening in cancer prevention.

Studies that have examined the impact of health fairs have favourably acknowledged health fairs as behaviour changing tools. According to Lucky et al. (2011), 93\% of people who were told they had high blood pressure at a health fair followed up with medical providers. ${ }^{7}$ Clark (1985) reported statistically significant improvements in mindset and knowledge at the conclusion of a fair following a pre-test, post-test study of health fair participants. ${ }^{8}$ Similarly, rural farmers who were surveyed months after a health fair reported positive changes as a result of information learned. Seventy eight percent of the farmers reported either work or lifestyle changes. ${ }^{9}$

Health fairs have also been found to be good informational tools among black Americans and non-white Hispanic populations. Participants at a black and minority health fair in Chicago were found to have significant changes in health knowledge and behaviour 15 months after the fair. ${ }^{10}$ A look at black Americans knowledge and attitude after a 1993 health fair also reported positive results. ${ }^{11}$ Additionally, a 2014 study by Murray et al. showed that health fairs serve as adequate tools to reach underserved 
Hispanics. ${ }^{1}$ Overall, these studies illustrate health fairs as appropriate tools to reach members of underserved and racial minority communities.

\section{Purpose}

While previous studies have looked at the effectiveness of health fairs in the setting of participant's subsequent knowledge or behaviour changes, the main objective of this study was to examine the effectiveness of health fairs in disseminating cancer screening information within target populations. Per the Centers for Disease Control and Prevention (CDC), early cancer screening for colorectal, lung, breast and cervical cancer saves lives. Obtaining maximal screening rates in burdened communities is of utmost importance. ${ }^{12}$ We aim to increase preventative screening rates of the underserved population and mitigate cancer mortality in these communities. Our hypothesis is that health fair participants will share the brochures and knowledge of screening recommendations received from health fairs with family and members of their social circle. It is our hopes that the preventative cancer screening information presented in this study will be used to inform the general public about the importance of health fairs and the need to disseminate information among members of the community.

\section{Materials and Methods}

Study data was collected at community health fairs in underserved minority communities of New York City in summer 2016. Staff members arrived at the health fair site at least 30 minutes prior to the beginning of the fair to set up the information table. A large table cloth with the Mount Sinai Hospital Tisch Cancer Institute logo was used to alert participants that medical information was available at the table. Materials on the table included a variety of pamphlets and brochures created by and ordered from the American Cancer Society. Brochure titles included Cancer Facts for Men, Think about Testing for HPV, Prostate Cancer, Breast Cancer and What Women Should Know about Cervical Cancer and HPV. Tables also contained giveaway gifts (mirrors, pens, pill boxes, reusable shopping bags) to attract participants. Participants were encouraged to take as many of each brochure as they wanted. Staff members were also available to discuss the brochures and screening recommendations. All brochures were printed in English and Spanish.

Each health fair table was staffed by at least one Mount Sinai Hospital Tisch Cancer Institute staff member and one volunteer, most of whom were cancer survivors representative of the underserved community. When patrons approached our tables which contained breast, cervical and colorectal cancer information, they were welcomed by staff members and volunteers, and directed to take materials that were of interest to them, with any questions they had answered as well. After participants got information they wanted, we introduced the research project and asked whether the participant would be interested in completing the survey.

Data was collected with a brief survey questionnaire that was given in-person at the health fair and by telephone 10 days after the health fair. Both in-person and follow-up interviews took approximately 5 minutes each. We did not use validated measures for the study. However, many of the questions used have been used in some of our previous studies and found to be reliable measures.

Institutional Review Board permission included waiver of signed consent.

\section{Participants}

From June 14, 2016 to August 26, 2016, tables providing information about cancer screening were established at 12 local community health fairs in New York City. All tables offered uniform health education information on breast, cervical, and colorectal cancer screening. Participants were 18 years or older and spoke English or Spanish. Those who agreed to participate were interviewed with a questionnaire that consisted of closed and open ended questions.

All questionnaires and study materials were translated into Spanish and staff members who were fluent in Spanish conducted surveys to participants who were Spanish Speaking only. In total, two in-person interviews were conducted in Spanish and both these Spanish speakers did not respond to follow up.

\section{Statistical analyses}

\section{Questionnaire}

The in-person questionnaire conducted at the health fairs contained three sections. Section 1 included demographic questions which have been used in our previous studies and found to be reliable, and inquired about: neighbourhood of residence, gender, age, marital status, highest education level, place of birth (if participant was not born in the United States, we asked how long they had lived in the United States), race and ethnicity.

Section 2 contained questions about participant's health care: we asked whether the participant had a regular healthcare provider, and whether the participant knew anyone who had cancer. We also asked Cancer Screening Behaviour questions that have been used in previous Mount Sinai Hospital Tisch Cancer Institute studies and found to be understood by participants. Men were asked about whether they have had a Digital Rectal Exam, Prostate Specific Antigen, and Colonoscopy. Women were asked about Clinical Breast Exam, Mammogram, Pap Exam, and Colonoscopy. For each of these tests, participants were asked whether they ever had the exam and if yes, the year of their last exam.

Section 3 included open ended questions that were developed for this study. We asked three questions: (1) What attracted you to the table?; (2) Were you interested in a specific cancer?; (3) Is there any information that you were seeking that we are missing?

There were two sections in the follow up questionnaire. Section 1 contained demographic questions which were identical to the questions asked in Section 1 of the initial interview, as the data from the initial interview at the health fair and the follow up phone interviews were not linked.

Section 2 contained questions that were developed for this study to gain insight into how participants used the information after the fair. The questions are below:

i. Do you recall stopping by the Mount Sinai Hospital Cancer prevention table?

ii. Did you read any of the cancer awareness material that was provided? If yes, how would you summarize the main points of the material?

iii. What did you like about the material you received? What did you not like about the material you received?

iv. Was there a specific pamphlet or information sheet that you really liked? If yes, which one?

v. Did you share the information with anymore?

vi. If information was shared: whom did you share the information with, why did you share the information with that specific person(s) and how did you share the information?

vii. Did receiving the information at the table make you want to talk to your doctor about cancer screening? 
viii. Since the health fair, have you spoken with your doctor about cancer screenings?

\section{Analyses}

Statistical analyses were conducted to evaluate what attracted participants to the health fair table, how well they remembered cancer screening information, and if participants disseminated cancer screening information received.

Open ended questions were categorized into themes for analysis. These open ended questions were coded into categories by two independent people and consensus on differences was then conducted. ${ }^{13}$ If a participant listed multiple answers, two independent people assessed which answer was more emphasized by the participant, and reached an agreement on which answer should be included for analysis. In performing analyses, we limited participant responses to one answer to make data analysis cleaner and easier to understand. For example, the question, Who did you share the information with? may have been many listed family members, which were coded as other family members, whereas, Daughter had its own category. We did not leave out any new information and only excluded redundant information. Statistical analyses were performed using the IBM SPSS 22.0.

\section{Results}

One hundred and sixteen participants participated in the initial survey. Of those, 88 participants (78\%) agreed to give their contact information for the follow up survey and 60 follow-up surveys were completed ( 3 participants declined to participate, 4 gave us wrong numbers and 21 participants were unable to be reached after numerous attempts.). As to those who read the material and recalled at least one detail, $45 \%$ shared the information with others; $15 \%$ subsequently spoke to a provider about cancer screenings and $40 \%$ intended to speak to a provider. Participants that shared the screening information did so with 1-3 people.

\section{Demographics}

Table 1 summarizes the demographic of the 116 participants who completed the in-person survey and the 60 participants who completed the follow-up telephone survey. There were no statistical differences in demographics between the two groups. Of the initial participants, the majority were single $(63 \%)$, identified as black/African American (76\%), and had completed high school or higher education (67\%). 21.5\% identified as Latino/Hispanic. Most participants had lived in the United States their entire life (76\%) and the rest had moved to the United States between 2-54 years ago.

Of participants who completed the follow-up survey, most identified as single (60\%), black/African American (70\%), and had completed high school or higher education (59\%). Only 17\% identified as Latino/Hispanic. Similar to the initial survey participants, most follow-up survey participants had lived in the United States their entire lives $(77 \%)$.

The majority of participants for both the initial and follow-up surveys were women ( $86 \%$ and $80 \%$ respectively).

\section{Participants' interest in our cancer prevention table}

Participants who approached our table reported three main reasons: seeking general information about cancer (42\%), just browsing the fair (20\%) and seeking free giveaways (7\%). Though most participants were seeking general cancer information (46\%), the majority of those who were seeking information on a specific can- cer mentioned an interest in learning more about breast cancer (27\%). When asked about missing information at our table, most participants felt that sufficient information was provided (84\%). Of the 116 participants who approached our table, 97\% reported having a regular healthcare provider and $90 \%$ reported knowing someone who had cancer (Table 2).

Table 3 summarizes the in-person open ended questions and the key themes used to code the information from the in-person and follow-up open ended questionnaires. Both questionnaires were categorized in a similar fashion. For example, for the followup open ended questionnaires, we looked at if participants could remember at least one point from the material: that is, 1. if a par-

Table 1. Demographic and background characteristics at time 1 (baseline) and time 2 (follow-up).

Characteristic In-person (N=116) Follow-up $(\mathrm{N}=60)$

\begin{tabular}{lcc} 
Female, $\%$ & 85 & 80 \\
Age, years & & \\
Mean \pm S.D. & $58.4 \pm 13.3$ & $53.87 \pm 12.2$ \\
Range & $18-95$ years & $24-74$ years \\
\hline Marital Status, \% & \\
$\quad$ Single & 63 & 60 \\
Married & 17 & 20 \\
Divorced & 7 & 10 \\
Widowed & 6 & 3 \\
Other & 7 & 7 \\
Ethnicity, $\%$ & & \\
Hispanic & 21 & 17 \\
Non-Hispanic & 79 & 83 \\
\hline Race, \% & & 70 \\
Black & 76 & 5 \\
White & 3 & 17 \\
Unknown & 15 & 8 \\
Other & 6 & \\
Education, \% & & 2 \\
Completed grade school only & 1 & 22 \\
Completed high school only & 29 & 15 \\
Completed college only & 21 & 22 \\
Completed post-grad & 17 & 40 \\
Other & 32 & 77 \\
Born in the US & 76 & \\
\hline
\end{tabular}

Table 2. Participant's baseline interest in our cancer prevention table.

\section{$\%$}

Why patrons approached table

General information about cancer $\quad 42.2$

Just browsing fair $\quad 19.8$

Free Giveaways $\quad 6.9$

Other 31.1

$\begin{array}{ll}\text { Patrons interest in specific cancer } & \\ \text { No specific cancer } & 45.6 \\ \text { Breast } & 26.2 \\ \text { Multiple } & 12.6 \\ \text { Other } & 15.6\end{array}$

Information lacking at table?

No information missing $\quad 83.8$

Another specific cancer (e.g. lung) 6.1

More screening options 5.1

$\begin{array}{ll}\text { Other } & 5.0\end{array}$ 
ticipant gave general positive response, but could not remember specific points 2. if a participant did not read material, and 3. if a participant could not remember material. Additionally, participants stated they liked the following themes regarding the material: good readability, exam picture diagrams, generally informative, easy to understand, or did not remember anything specific. What participants did not like about the material was mostly divided between: they did not dislike anything or they would like more information about other cancers. Why participants shared information was categorized into normal practice, they thought it would be generally informative for another person, or the person they shared the information with has/had cancer or knew someone who has/had cancer. If the participant shared the screening information, the number of people and the relationship to the participant was also recorded. Participants shared information by giving out pamphlets, speaking with someone about the information, or both.

\section{Cancer screening adherence}

Adherence was computed using the CDC recommended guidelines for screening. ${ }^{14}$ Among men, 64\% were adherent for colonoscopy. For women, adherence rates were $84 \%$ for mammography, $84 \%$ for pap smear, and $83 \%$ for colonoscopy. Table 4 contains screening adherence information for participants.

For the purposes of our study, participants who were unable to recall screenings done every 2 to 3 years (i.e. mammogram and/or pap smear) were marked as non-adherent given the relatively short time frame. As the time frame is longer for colonoscopy adherence (10 years), participants who were unable to remember their last colonoscopy were removed from the sampling pool when analysing for adherence. We made the assumption that participants would remember a procedure they had done within the past 2-3 years. However, since the generally recommended screening interval for colonoscopy is 10 years, we assumed that it may be easier to forget whether one had the procedure done within this wider time frame.

\section{Demographic and behavioural patterns of screening adherence rates}

Adherence to colonoscopy, mammography, and cervical cancer screenings was compared to various metrics: why participants approached the table, race, ethnicity, and education level, whether participants had a regular healthcare provider, whether a participant knew someone with cancer, and whether participants were interested in learning about a specific cancer. Please see Table 4 for reference.

Overall, participants who reported knowing someone with cancer showed increased cancer screening adherence with the exception of mammography ( $85 \%$ participants who knew someone with cancer were adherent to mammography compared to $89 \%$ mammography adherence in women who did not know anyone with cancer). For cervical cancer screening and female colonoscopy screening, we observed higher adherence rates for participants who did not report having a regular healthcare provider.

Participants whose highest level of education was high school had the highest screening adherence rates for all cancers $(88 \%$ for female colonoscopy, $100 \%$ for male colonoscopy, and $90 \%$ for pap smear) except mammography, in which those with some college education had the highest screening rates (90\%). Of African American women, $84 \%$ were up to date on colonoscopy, $86 \%$ are up to date on mammography and $90 \%$ were up to date on pap tests.

Table 3. Baseline in-person open ended question themes and follow-up open ended question themes.

\begin{tabular}{|c|c|}
\hline \multicolumn{2}{|c|}{$\begin{array}{l}\text { Question Theme } \\
\qquad \text { In-Person Open Ended Questions }\end{array}$} \\
\hline What attracted you to the table? & $\begin{array}{l}\text { I have/had cancer; I know someone who has/had cancer; General information } \\
\text { about cancer; Just walking through the health fair; Wanted free giveaways; } \\
\text { Mount Sinai logo; Color of the table; More than one of the categorized reasons }\end{array}$ \\
\hline Were you interested in a specific cancer? & $\begin{array}{l}\text { No / (Yes - specific type); Breast; Cervical; Colorectal; Prostate; Lung; Uterine; } \\
\text { Stomach; Skin; Multiple specific types }\end{array}$ \\
\hline Is there any information that you were seeking that we are missing? & $\begin{array}{l}\text { Yes - another cancer (e.g, lung cancer); Yes - another non-cancer disease } \\
\text { (e.g, diabetes); No; Support groups; Progress in curing cancer }\end{array}$ \\
\hline Follow- & en Ended Questions \\
\hline
\end{tabular}

How would you summarize the points of the materials?

points;

What did you like about the material that you received?

Was there a specific pamphlet or information sheet that you really liked? If yes, which one (s)?

What did you not like about the material that you received?

Was the information shared?

Who did you share the information with?

Why?

How did you share the information?
Participant could remember at least 1 point from the material; Participant gave general positive response (e.g, informative), but did not remember specific

Participant did not read the material; Participant could not remember material Good readability; Exam picture diagrams (e.g, Self Breast Exam); Informative; Easy to understand; Does not remember anything specific

No; Breast cancer; Self-breast exam; Cancers in men

Did not dislike anything; Would like more information about other cancers; Too wordy; Did not remember

No / Yes

Wife; Husband; Daughter; Son; Sibling; Mom; Dad; Other family member(s); Friends(s); Coworkers(s); Neighbors(s); Fiancé(e); Strangers

Normal practice; Thought it would be generally informative for another person; The person they shared with has/had cancer; The person they shared with knows someone who has/had cancer

Gave them the pamphlet; Spoke with them about the information; Both 


\section{Sharing information received}

As reported by the follow up participants, $45 \%$ reported sharing the information they received at the health fair (Tables 5 and 6 summarizes sharing behaviours). Participants who read the brochure $(60 \%)$ shared the information more than those who did not $(11 \%)(\mathrm{P}<0.001)$. Participants who could summarize at least one point from the material $(72 \%)$ shared the information more often than those who could not $(50 \%)$. Men, though a small sample, shared the information more than women $(58 \%$ men versus $41 \%$ women). Married participants were more likely to share $(58 \%)$ than single (44\%) and divorced (16\%) participants. There were minor differences based on education; $50 \%$ of participants who had completed high school and some college, and $45.5 \%$ of participants who had completed college or post graduate studies shared the information.

Participants who thought the information might be informative to another person (59\%) were more likely to share it. The information received was mostly shared by giving out the brochures $(33 \%)$, telling others about the information $(30 \%)$, or doing both $(37 \%)$. Information was more likely shared among close friends and family (78\%) than with co-workers and strangers $(11 \%)$.

\section{Discussion}

Providing cancer screening information to members of low income and racial minority groups is important as these groups bear a large cancer mortality burden. This study found that health fair participants who read cancer prevention material shared the information with other members of underserved communities that were not present at the fair at statistically significant rates. Overall, information was disseminated without prompting, suggesting the reach of healthcare fairs extends beyond the people who visit the fair. The results of our study revealed statistically significant differences in sharing information between individuals who read the brochure and those who did not $(\mathrm{P}<0.001)$. Individuals who read the brochure and found it to be informative were more likely to share than those who did not. This allowed for information to be propagated to others in their individual communities. More resources should therefore be put towards educating individuals from underserved communities to help augment the cancer screening gap that exists in underserved communities.

While the cancer screening gap between black, Hispanic and white Americans has been reduced for most cancers, there exists a strong association between education, income, screening rates, and outcomes. ${ }^{15,16}$ Data from 1988-2015 shows that black Americans

Table 4. Baseline cancer screening adherence rates: demographic and behavioral patterns.

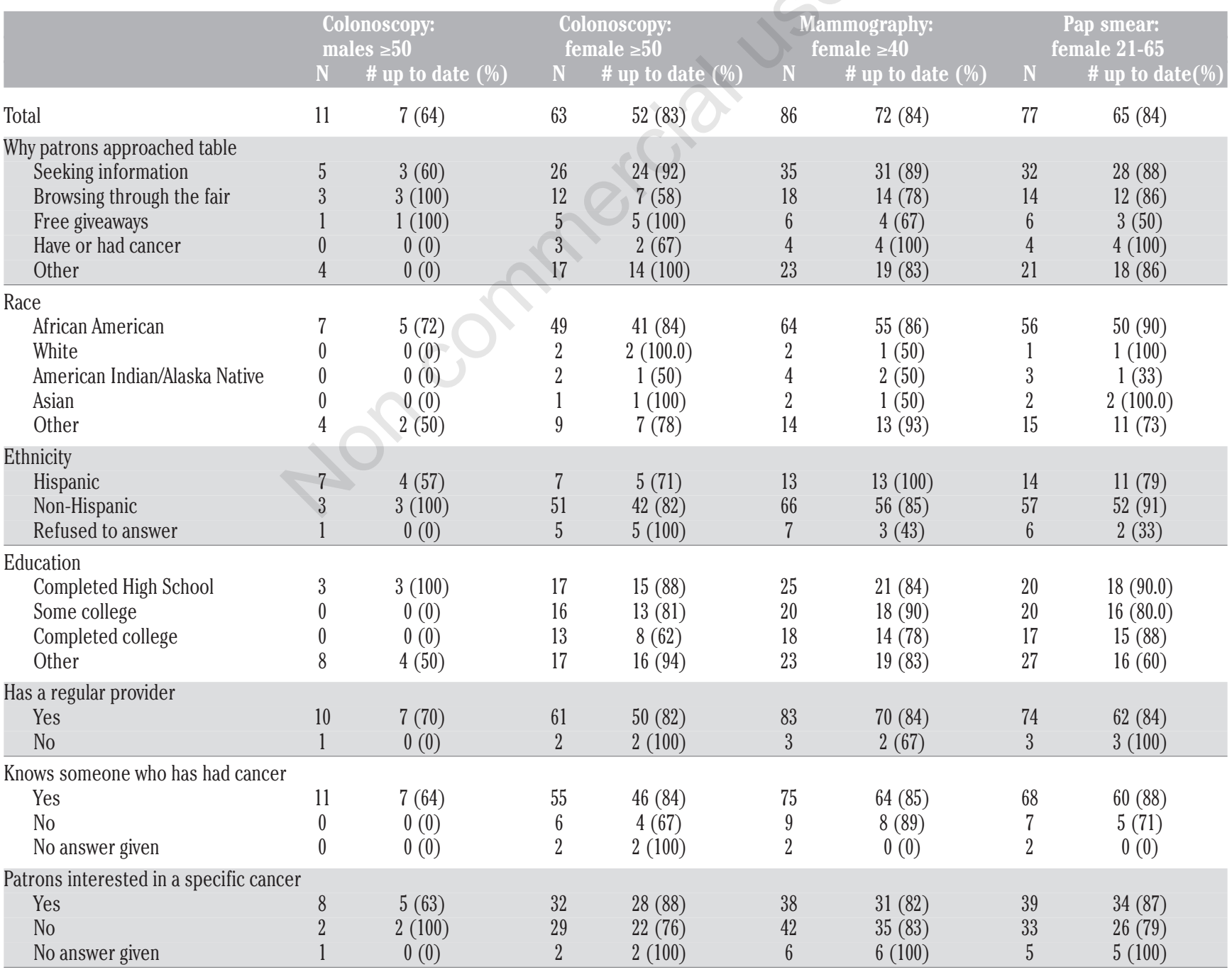

Adherence $=$ Yes (and within recommended timeframe); Colonoscopy: every 10 years from 50 years and older; 3 excluded for missing dates; Clinical breast exam: every one to three years starting at age 20 and every year starting at age $40^{*}$; Mammograms: every 2 years for women age 40 to $75^{*}$; Pap smear: every three years for women ages 21 to $65^{*}$. ${ }^{*}$ Women who did not remember year of screening test marked as non-adherent. 
have consistently lagged by $11-14 \%$ in college degree attainment behind their non-Hispanic white peers, and Hispanics lag behind black Americans. ${ }^{17}$ Black and Hispanic Americans are more often impoverished (poverty rate of $26 \%$ for blacks $v$ s. $10 \%$ for nonHispanic whites) and are more likely to live in isolated poverty neighbourhoods, which sociologists believe leads them to experience a double burden of poverty. ${ }^{18,19}$ In New York City, six out of the seven districts with concentrated poverty rates of greater than $50 \%$ are comprised of black or Hispanic populations. ${ }^{20}$ The strong association between education level/income and screening adherence suggests a need for continued health screening education in low income and minority neighbourhoods. And with increased mortality rates in these communities, one might argue that screening rates need to be highest in these communities, in addition to improving access to follow-up care.

Although closing knowledge gaps in preventative screening recommendations is a key barrier to care in underserved communities, other barriers must be overcome in order to improve cancer mortality and the mortality disparity between underprivileged groups and their more privileged peers. For example, despite equivalent breast cancer screening rates, black women continue to die at higher rates from breast cancer than white women due to factors such as delayed diagnosis and treatment: According to the C.D.C., even when they have similar insurance coverage, $20 \%$ of black women with an abnormal mammogram wait more than 60 days for a diagnosis, compared with 12 percent of white women. And 31 percent of black women wait 30 days to begin treatment, compared with 18 percent of white women. ${ }^{2}$ Teaching patients to be better advocates for themselves once they have entered into the healthcare system demonstrates a potential future focus for health fair prevention tables in underserved communities.

Using the demographic information collected (even though we did not have information for all participants that visited the fair), it was unfortunate to see that few black men visited our cancer prevention table given the dismal statistics for black men in regards to cancer: black men have both the highest cancer incidence and mortality rates in this country. ${ }^{21}$ Black men who participated however showed a higher propensity to share information than their female counterparts. The lack of men may be tied to the expanded role of black women as providers and caretakers in their communities: in nearly $44 \%$ of black families with children, a woman is the primary breadwinner. This includes both families headed by working single mothers and married-couple families in which the wife works and the husband does not.... In contrast, across all racial and ethnic groups, female breadwinner families represent only 24\% of all families with children. ${ }^{22}$ Unlike women in many other communities, black women play a pivotal role in supporting their families. Still, given the mortality burden of black men in the U.S, it is important for future studies to find appropriate ways to engage them and foster healthcare awareness.

There were several limitations to this study. First, while past research supports the use of self-reporting, using self-reported surveys to assess cancer screening adherence in our study may not accurately reflect screening behavioural patterns of participants. ${ }^{23-}$ ${ }^{25}$ Participants may incorrectly remember screening dates and/or be subject to social desirability bias, in which responses are tailored in a way interviewees believe will be viewed favourably by the interviewer. Promisingly, the reported screening rates met or exceeded the Healthy People 2020 goals, with the exception of pap smear and male colonoscopy (mammogram $81.1 \%$, cervical $93 \%$, colonoscopy, $70.5 \%)^{26}$

Additionally, high screening rates among participants of this study may also be because those with prior experience of the perils of cancer could have had a higher interest in approaching a table with preventative cancer information. We attempted to attract other health fair participants without cancer specific experience by offering free giveaways such as reusable shopping bags, pens, pill holders, mirrors, t-shirts, and hats.

Also, the educational attainment of our sample also exceeds national averages. For example, $17.4 \%$ of our sample reported completing advanced post-graduate studies, while the national rate of black and Hispanic Americans who have completed advanced post-graduate work is $8.2 \%$ and $4.7 \%$ respectively. This may be due to the health fair's location in New York City, a large

Table 5. Follow-up questionnaire responses.

\begin{tabular}{|c|c|c|}
\hline $\begin{array}{l}\text { Read information? } \\
\text { Yes }(\mathrm{N}=42) \\
\text { No }(\mathrm{N}=18)\end{array}$ & $\begin{array}{c}25(60) \\
2(11)\end{array}$ & $\begin{array}{l}17(41) \\
16(89)\end{array}$ \\
\hline $\begin{array}{l}\text { Summarize at least one point from material? } \\
\text { Yes }(\mathrm{N}=18) \\
\text { No }(\mathrm{N}=24)\end{array}$ & $\begin{array}{l}13(72) \\
12(50)\end{array}$ & $\begin{array}{c}5(28) \\
12(50)\end{array}$ \\
\hline $\begin{array}{l}\text { Reports liking a specific pamphlet or brochure } \\
\text { Yes }(\mathrm{N}=7) \\
\text { No }(\mathrm{N}=35)\end{array}$ & $\begin{array}{c}5(71) \\
20(57)\end{array}$ & $\begin{array}{c}2(29) \\
15(43)\end{array}$ \\
\hline $\begin{array}{l}\text { Gender } \\
\text { Male }(\mathrm{N}=12) \\
\text { Female }(\mathrm{N}=48)\end{array}$ & $\begin{array}{c}7(58) \\
20(42)\end{array}$ & $\begin{array}{c}5(42) \\
28(58)\end{array}$ \\
\hline $\begin{array}{l}\text { Marital Status } \\
\text { Single }(\mathrm{N}=36) \\
\text { Married }(\mathrm{N}=12) \\
\text { Divorced }(\mathrm{N}=6) \\
\text { Other }(\mathrm{N}=6)\end{array}$ & $\begin{array}{l}16(44) \\
7(59) \\
1(17) \\
3(50)\end{array}$ & $\begin{array}{l}20(56) \\
5(42) \\
5(83) \\
3(50)\end{array}$ \\
\hline $\begin{array}{l}\text { Education Level } \\
\text { Completed HS }(\mathrm{N}=12) \\
\text { Some College }(\mathrm{N}=16) \\
\text { Completed College or Post-Grad }(\mathrm{N}=22) \\
\text { Other }(\mathrm{N}=9)\end{array}$ & $\begin{array}{l}6(50) \\
8(50) \\
10(46) \\
5(56)\end{array}$ & $\begin{array}{l}6(50) \\
8(50) \\
12(54) \\
4(44)\end{array}$ \\
\hline
\end{tabular}


metropolitan area, even though these health fairs were conducted in low income neighbourhoods. Future health fairs could tease out this potential bias by conducting fairs in rural areas or in closer proximity to specific government housing projects in order to reach a greater number of underserved people with the demographics of our target audience.

Since our enrolment was conducted via a convenience sample that was racially homogenous and in a very specific urban setting, results may not be generalizable to other populations. In addition, health fair productions are unregulated and unstandardized, and though our table had the same set-up for the different fairs, the ambience of each fair was different leading to external factors that potentially affected participant's participation or recruitment - for example, at a few fairs, the music was loud, impeding the ability to conduct surveys.

Finally, the attrition rate of the people who agreed to the follow up could be seen as a set-back to our study. There may be systematic differences between the participants lost to follow-up and those who responded. Despite these limitations, our study was able to analyse the effectiveness of health fairs in disseminating cancer screening information.

\section{Conclusions}

In conclusion, this paper demonstrates the effectiveness of healthcare fairs in spreading cancer screening information among minority and underserved populations (i.e., low income and racial minority groups). When addressing cancer mortality, social determinants of health (especially education and economic stability) cannot be ignored and a close look at these social determinants should yield a more thorough understanding of how to reduce mortality in low income and racial minority communities. However since early detection is known to save lives, it is important that we continue to find ways to advance cancer prevention education in these communities. Future studies should investigate whether participants will share information at higher rates when they are explicitly encouraged to distribute the information.

This project was fairly easy to implement at a low cost to organizers. As these health fairs were open for people to freely enter

Table 6. Follow-up distribution of preventative screening material.

\begin{tabular}{lc}
\hline Question & $\%$ \\
What patrons who read and shared liked about brochures? (N=25) & \\
$\quad$ Informative & 60 \\
Easy to understand & 17 \\
Good readability & 10 \\
Why people shared information? (N=27) & \\
Thought it would be informative for another person & 59 \\
$\quad$ Normal practice & 19 \\
Person they shared it with has/had cancer & 11 \\
Person they shared it with knows someone who has/had cancer & 11 \\
\hline How did they share it? (N=27) & \\
Gave pamphlet & 33 \\
Spoke to person about information & 30 \\
Did both & 37 \\
Shared with whom? (N=27) & \\
With family and friends (husband, daughter, siblings) & 78 (7.4) \\
Co-workers & 11 \\
Strangers & 11 \\
\hline
\end{tabular}

and leave, education and socioeconomic status were not barriers to participation. Future health fairs could also highlight access to more educational programs and on-site screenings to further enhance patient care in underserved communities.

Correspondence: Erica Monrose, Icahn School of Medicine of Mount Sinai, 1 Gustave L. Levy Place, New York, NY 10029-5674, USA.

Tel.: + 1.646.436.5225.

E-mail: erica.monrose@icahn.mssm.edu

Key words: cancer prevention; outreach; health fairs.

Contributions: EM, conception or design of the work, data collection, data analysis and interpretation, drafting the article; JL, data collection, data analysis and interpretation, drafting the article, critical revision of the article; DA, data analysis and interpretation, drafting the article; LJ, conception or design of the work, data analysis and interpretation, drafting the article, critical revision of the article. All authors approved the final version of the paper.

Conflict of interest: the authors declare no conflict of interest.

Received for publication:10 March 2017.

Accepted for publication: 30 August 2017.

(C) Copyright E. Monrose et al., 2017

Licensee PAGEPress, Italy

Journal of Public Health Research 2017;6:866

doi:10.4081/jphr.2017.866

This work is licensed under a Creative Commons Attribution NonCommercial 4.0 License (CC BY-NC 4.0).

\section{References}

1. Murray K, Liang A, Barnack-Tavlaris J, Navarro AM. The reach and rationale for community health fairs. J Cancer Educ 2014;29:19-24.

2. Freeman HP. Why black women die of cancer. The New York Times 2014-1.

3. O'Keefe EB, Meltzer JP, Bethea TN. Health disparities and cancer: racial disparities in cancer mortality in the United States, 2000-2010. Front Public Health 2015;3:51.

4. Siegel RL, Fedewa SA, Miller KD, et al. Cancer statistics for Hispanics/Latinos, 2015. CA Cancer J Clin 2015;65:457-80.

5. Heron M. Deaths: leading causes for 2014. Natl Vital Stat Rep 2016;65:1-96.

6. Conway J. Disparities in health care: the black population. Oncol Nurse Adv 2012:22-9.

7. Lucky D, Turner B, Hall M, et al. Blood pressure screenings through community nursing health fairs: motivating individuals to seek health care follow-up. J Commun Health Nurs 2011;28:119-29.

8. Clark MJ. The health fair: an effective approach to health promotion? Public Health Nurs 1985;2:33-42.

9. Rydholm L, Kirkhorn SR. A study of the impact and efficacy of health fairs for farmers. J Agric Saf Health 2005;11:441-8.

10. Seo DC. Lessons learned from a black and minority health fair's 15-month follow-up counseling. J Natl Med Assoc 2011;103:897-906.

11. Brown CM, Khan ZM. A survey of African Americans at a community health fair. J Health Care Poor Underserved 1998;9:357-66.

12. Lawson HW, Henson R, Bobo JK, Kaeser MK. Implementing recommendations for the early detection of breast and cervical cancer among low-income women. Oncology (Williston Park) 2000;14:1528-30. 
13. Bryman A, Burgess B, eds. Analyzing qualitative data. New York: Taylor and Francis; 1994.

14. US Preventive Services Task Force Guides to Clinical Preventive Services. the guide to clinical preventive services 2014: recommendations of the U.S. preventive services task force. Rockville: US Preventive Services Task Force Guides to Clinical Preventive Services; 2014.

15. Health, United States, 2014: With Special Feature on Adults Aged 55-64. Rockville MD 2015. Available from: https://www.cdc.gov/nchs/data/hus/hus14.pdf

16. Bradley CJ, Given CW, Roberts C. Race, socioeconomic status, and breast cancer treatment and survival. J Natl Cancer Inst 2002;94:490-6.

17. Ryan CL, Bauman K. Educational attainment in the United States: 2015. U.S Census Bureau, U.S. Department of Commerce: Economics and Statistics Administration; 2015. Available

from: https://www.census.gov/content/dam/Census/library/publications/2016/demo/p20-578.pdf

18. Proctor BD, Semega JL, Kollar MA. Income and poverty in the United States: 2015. In: Administration USDoCEaS. Washington D.C.: U.S Census Bureau; 2015.

19. Jargowsky P. Architecture of segregation: civil unrest, the concentration of poverty, and public policy. The Century Foundation; 2015.

20. Citizens' Committee for Children. Concentrated Poverty in New York City. April 2012. Available from: https://www.ccc-
newyork.org/wp-content/publications/CCCReport. ConcentratedPoverty.April-2012.pdf

21. American Cancer Society. Cancer facts and figures for African Americans 2016-2018. Atlanta, GA: American Cancer Society, Inc.; 2017. Available from: https://www.cancer.org/content /dam/cancer-org/research/cancer-facts-and-statistics/cancerfacts-and-figures-for-african-americans/cancer-facts-and-figures-for-african-americans-2016-2018.pdf

22. Conrad CA. Black women: the unfinished agenda. African American women made great progress in education and entering into previously forbidden occupations-but their gains in earnings mysteriously stopped. The American Prospect 2008:19.

23. Kandula NR, Wen M, Jacobs EA, Lauderdale DS. Low rates of colorectal, cervical, and breast cancer screening in Asian Americans compared with Non-Hispanic whites: cultural influences or access to care? Am Cancer Soc 2006;107:190.

24. Wu TY, West B, Chen YW, Hergert C. Health beliefs and practices related to breast cancer screening in Filipino, Chinese and Asian-Indian women. Cancer Detect Prev 2005;30:58-66.

25. Gomez SL, Tan S, Keegan TH, Clarke CA. Disparities in mammographic screening for Asian women in California: a cross-sectional analysis to identify meaningful groups for targeted intervention. BMC Cancer 2007;7:201.

26. National Center for Health Statistics. Cancer Trends Progress Report. Breast Cancer Screening: National Center for Health Statistics; 2017. 